\title{
Characterization of an Improved, Real-Time MEMS-Based Phase-Shifting Interferometer
}

\author{
R Kant D Garmire H C oo and R S Muller \\ Department of Electrical Engineering Stanford University Stanford CA USA \\ 7X Allen CIS Building Extension 4 Via Palou Mall Stanford CA USA 945 \\ Fax 958476678 E mail rik9@stanford edu \\ Berkeley Sensor Actuator Center University of California Berkeley CA USA
}

\begin{abstract}
We describe and present detailed performance c aracterizations for an en anced version of our MEMS Based P ase S ifting Interferometer MBPSI) $t$ at ac ieves times denser motion reconstruction $t$ an our original system We measure $t$ e noise level to be $\leq \pm 6 \mathrm{~nm} \lambda$ for a $66 \mathrm{~nm}$ laser) and $\mathrm{t}$ e frequency resolution to be $\leq \mathrm{Hz}$ for $\mathrm{Hz}$ motion captured at $\mathrm{Hz}$ We ave successfully tracked a piezo based actuator driven wit an arbitrary waveform composed of transients $\leq \mathrm{Hz}$
\end{abstract}

Keywords: optical MEMS, Phase-shifting, interferometer, transient motion

\section{INTRODUCTION}

We ave greatly increased $t$ e motion reconstruction resolution of our previously reported MEMS Based $\mathrm{P}$ ase $\mathrm{S}$ ifting Interferometer MBPSI) ] from $\mathrm{Hz}$ to $\mathrm{Hz}$ $\mathrm{T}$ is improvement enables $\mathrm{t}$ e reconstruction of real time motion wit times more samples resulting in more precise measurements $\mathrm{T}$ e improvement is ac ieved $t$ roug a combination of ig er frame rate of $t$ e CMOS imager and newly developed post processing routines

Our MBPSI uses a resonating MEMS mirror as $\mathrm{t} e$ $\mathrm{p}$ ase $\mathrm{s}$ ifting element in conjunction wit a strobing laser as s own in Figure ] Sync ronizing t e strobes wit $t$ e resonating element eliminates $t$ e settling time delay typically found in conventional $\mathrm{p}$ ase $\mathrm{s}$ ifting interferometers allowing our MBPSI to ac ieve camera frame rate limited captures similar to digital olograp ic microscopy ] In addition to employing faster imager rates increase from 9 to $\mathrm{Hz}$ ) we utilize a simple yet ig ly effective new post processing algorit $\mathrm{m}$ described in Figure $w$ ic quadruples $t$ e time resolution of measurements

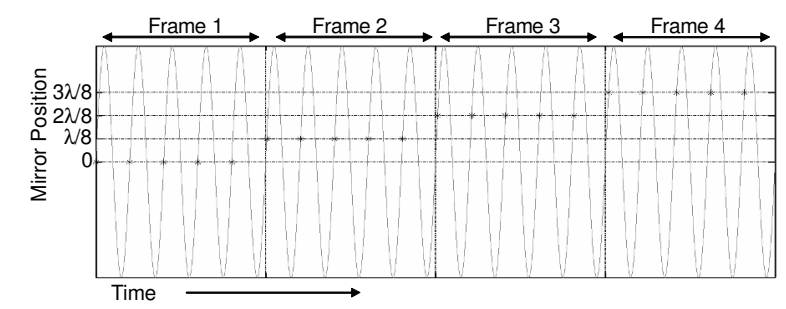

Figure 1. T e laser is strobed in sync denoted by " ) wit $t$ e resonating MEMS mirror to generate $t$ e four distinct $p$ ase $s$ ifts required for surface reconstruction wit in four camera frames

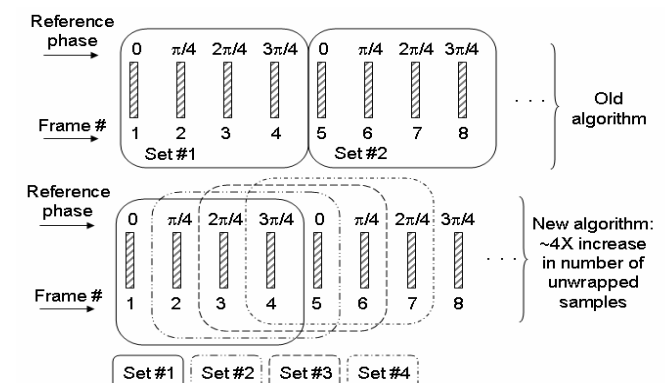

Figure 2. Our new post processing routine divides images into sets for reconstruction by using images from $t$ e previous set plus $t$ e next image yielding a $4 \mathrm{X}$ increase in $\mathrm{t}$ e number of reconstructed points

We ave also c aracterized $t$ e capabilities of $t$ e system by using a piezo bending actuator as $\mathrm{t}$ e test sample $\mathrm{T}$ e noise level is $\leq \pm 6 \mathrm{~nm} \lambda$ for a $66 \mathrm{~nm}$ laser) and $\mathrm{t} e$ frequency resolution of $\mathrm{t} e$ measurement is better $\mathrm{t}$ an

$\mathrm{Hz}$ for tracking $\mathrm{Hz}$ motions captured at $\mathrm{Hz}$ Finally we ave successfully applied our system to reconstruct arbitrary non periodic motions of a PZT actuator

\section{EXPERIMENT SETUP}

We use a modified Twyman Green configuration interferometer as $\mathrm{s}$ own in Figure $\mathrm{T}$ e test sample is a mirror mounted on a piezo driven bending actuator

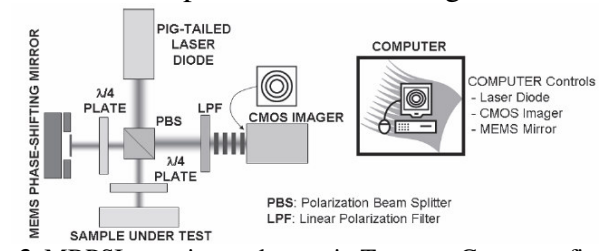

Figure 3. MBPSI experimental setup in Twyman Green configuration 
T e image area was set to 6 by 64 pixels corresponding to a $6 \mu \mathrm{m}$ by $64 \mu \mathrm{m}$ mirror surface $\mathrm{T}$ e camera gain and brig tness were constant for all experiments $\mathrm{T}$ e laser pulse widt was set to $\mu \mathrm{s} \mathrm{T}$ e laser strobes per frame were 8 and 5 for frame rates of and $\mathrm{Hz}$ respectively Every experiment was conducted wit in a capture window of 4 seconds Data were processed using t e 4 frame $\mathrm{p}$ ase unwrapping algorit $\mathrm{m} \mathrm{T}$ e position at eac point in time was calculated as $t$ e mean over $t$ e entire imaged area $\mathrm{T}$ e motion plots were generated by subtracting $\mathrm{t}$ e initial position from eac of $\mathrm{t}$ e subsequent positions

\section{RESULTS}

\subsection{Noise level}

$\mathrm{T}$ e noise level was determined by continuously measuring a static surface over 4 seconds and analyzing t e measured position variations $\mathrm{T}$ e istograms of position variations s ow Gaussian distribution wit standard deviation $\sigma$ ) of $47 \mathrm{~nm} \quad 5$ and $9 \mathrm{~nm}$ for and $\mathrm{Hz}$ frame rates respectively For Gaussian distribution 95 of $\mathrm{t} e$ values fall wit in $\pm \sigma$ of $t$ e mean value yielding a noise limit of $\leq \pm 6 \mathrm{~nm}$ for $\mathrm{t}$ e system $\pm \lambda$ for $66 \mathrm{~nm}$ laser) at

$\mathrm{Hz}$ Hig er frame rates s ow ig er noise levels because $\mathrm{t}$ e number of laser strobes decreases resulting in a lower signal to noise ratio of $t$ e captured images
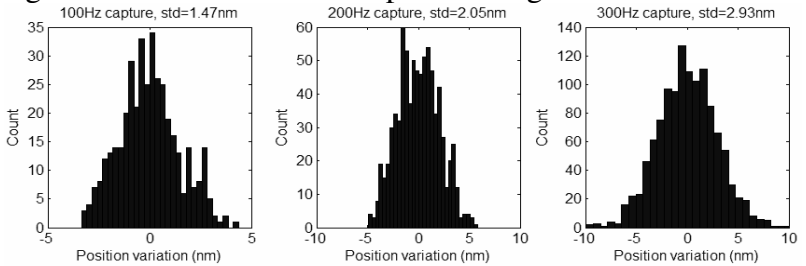

Figure 4 Measured position variation of a static surface over time at different frame rates yields a base noise level of $6 \mathrm{~nm} \quad \sigma$ ) for $\quad \mathrm{Hz}$

\subsection{Frequency-resolution}

To determine $\mathrm{t}$ e frequency resolution $\mathrm{t}$ e test sample over a $\pm 75 \mathrm{~nm}$ range wit sinusoids at known frequencies Analysis of $\mathrm{t} e$ frequency spectrum $\mathrm{s}$ ows $\mathrm{t}$ at $\mathrm{Hz}$ motion can be tracked to wit in $\mathrm{Hz}$ as s own in Figure 5

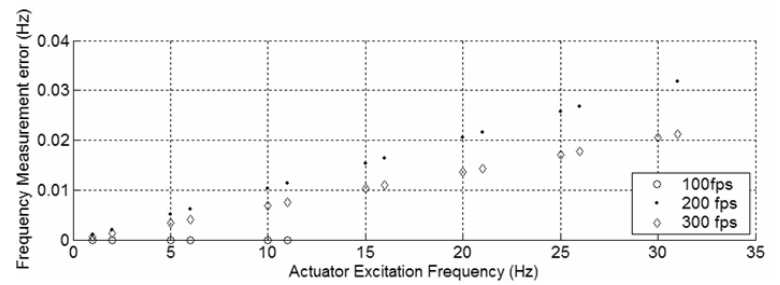

Figure 5 Frequency resolution of a moving actuator at different capture rates $\mathrm{s}$ ow $\mathrm{t}$ e expected trend of decreasing accuracy wit increasing sample frequency and increasing accuracy wit ig er capture rates

\subsection{Transient-motion capture}

Arbitrary transient motion was emulated by driving $t \mathrm{e}$ sample wit a custom waveform composed of five weig ted sinusoids in $\mathrm{t}$ e range of to $\mathrm{Hz}$ listed in Table )

Table 1 Frequency and weig ts of sinusoids used to generate arbitrary driving waveform

driving waveform
\begin{tabular}{|c|c|c|c|c|c|}
\hline & $\mathrm{f}$ & $\mathrm{f}$ & $\mathrm{f}$ & $\mathrm{f}_{4}$ & $\mathrm{f}_{5}$ \\
\hline Freq & $\mathrm{Hz}$ & $\mathrm{Hz}$ & $\mathrm{Hz}$ & $6 \mathrm{~Hz}$ & $\mathrm{~Hz}$ \\
\hline Weig t & 6 & 6 & 8 & 4 & \\
\hline
\end{tabular}

$\mathrm{T}$ e reconstructed motion closely matc es $\mathrm{t}$ e expected motion in $\mathrm{t}$ e time domain as $\mathrm{s}$ own by Figure 6 a) $\mathrm{T}$ e frequency spectrum plotted in Figure 6 b) $s$ ows $t$ at $t e$ largest deviation between expected and reconstructed motion arises from tracking $\mathrm{t}$ e amplitude of $\mathrm{t} e \mathrm{~Hz}$ component $\mathrm{T}$ e amplitude error is lower $\mathrm{w}$ en $\mathrm{t}$ e measurement rate is

$\mathrm{Hz}$ as expected
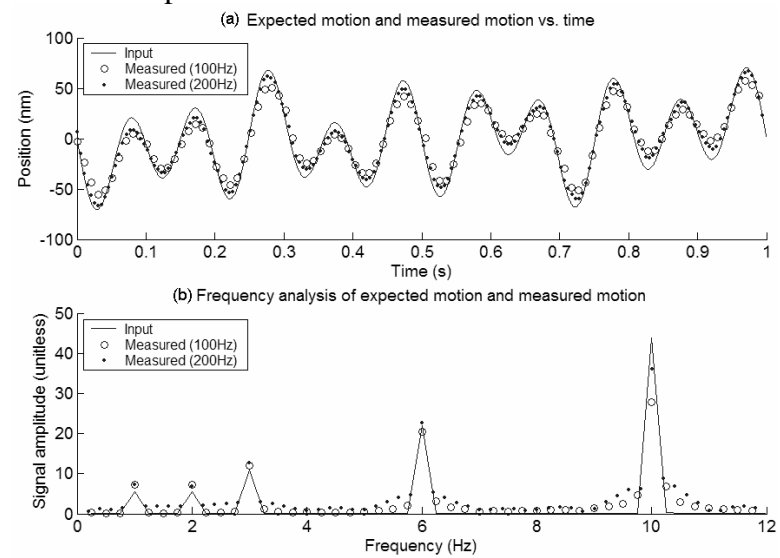

Figure 6 a) Expected and measured motion b) Frequency decomposition of expected and measured motion

\section{CONCLUSIONS}

We ave successfully demonstrated and carefully $c$ aracterized $t$ e improved version of our MEMS Based $\mathrm{P}$ ase $\mathrm{S}$ ifting Interferometer for real time transient $\mathrm{p}$ ase $\mathrm{s}$ ifting interferometry We obtain a noise level $\leq \pm 6 \mathrm{~nm}$ and frequency resolution of $8 \mathrm{~Hz}$ for tracking

$\mathrm{Hz}$ motions allowing us to accurately track $\mathrm{t}$ e real time motion of a piezo bending actuator

\section{REFERENCES}

] $\mathrm{H} \mathrm{C}$ oo R Kant D Garmire J Demmel and R S Muller Fast MEMS Based $\mathrm{P}$ ase $\mathrm{S}$ ifting Interferometer Proc of Solid State Sensors Actuators and Microwave Systems Hilton Head NC USA June $48 \quad 6$ pp 9495

] Y Emery E Cuc e F Marquet et al "Digital Holograp ic Microscopy DHM) for metrology and dynamic c aracterization of MEMS and MOEMS" Proceedings of SPIE April $6 \mathrm{pp}$ 59 